\title{
Smartphone-based reminder system to promote pelvic floor muscle training for the management of postnatal urinary incontinence: historical control study with propensity score- matched analysis
}

\author{
Kaori Kinouchi ${ }^{\text {Corresp., }}{ }^{1}$, Kazutomo Ohashi ${ }^{1}$ \\ ${ }^{1}$ Department of Children and Women's Health, Osaka University Graduate School of Medicine, Suita, Osaka, Japan \\ Corresponding Author: Kaori Kinouchi \\ Email address: kinouchi@sahs.med.osaka-u.ac.jp
}

Background. The purpose of this study was to evaluate the efficacy of a smartphonebased reminder system in promoting pelvic floor muscle training (PFMT) to help postpartum women manage urinary incontinence (UI). Methods. Forty-nine and 212 postpartum women in the intervention and control groups, respectively, received PFMT guidance using a leaflet and verbal instruction as the standard care at an obstetrics clinic in Japan. Women in the intervention group also received PFMT support using the smartphone-based reminder system between January and August 2014. For analysis, they were compared with historical controls between February 2011 and January 2012, who did not receive such support and were chosen by propensity score matching. The outcomes examined were PFMT adherence and UI prevalence. The former consisted of implementation rate (i.e., the percentage of women who reported performing PFMT during the intervention period), training intensity (i.e., the number of pelvic floor muscle contractions (PFMCS) per day), and training frequency (i.e., the number of days PFMT was performed per week); the latter consisted of self-reported UI prevalence at baseline and at the end of the 8-week intervention period. Result. Propensity score matching resulted in 58 postpartum women ( $n=29$ per group). The intervention group exhibited better PFMT adherence than the control group, in terms of PFMT implementation rate (69 vs. $31 \%, p=$ 0.008 ), median training intensity (15 vs. 1 PFMC reps/day, $p=0.006$ ), and training frequency ( 7 vs. 3 days/week, $p<0.001$ ). Ul prevalence was not different between the groups at baseline, but was significantly reduced in the intervention group at 8 weeks ( 0 vs. $24 \%, p=0.004)$. Conclusion. Our smartphone-based reminder system appears promising in enhancing PFMT adherence and managing postpartum UI in postpartum women. By enhancing PFMT adherence and improving women's ability to manage the condition, the reminder system could improve the health-related quality of life of postpartum women with UI. 
1 Smartphone-based reminder system to promote pelvic floor muscle training for the

2 management of postnatal urinary incontinence: historical control study with propensity

3 score-matched analysis

4

5 Kaori Kinouchi, Kazutomo Ohashi

6 Department of Children and Women's Health, Osaka University Graduate School of Medicine

7 Suita, Osaka, Japan

8

9 Corresponding Author:

10 Kaori Kinouchi

11 1-7 Yamadaoka, Suita, Osaka 565-0871, Japan

12 Email address: kinouchi@sahs.med.osaka-u.ac.jp 


\section{Abstract}

14 Background. The purpose of this study was to evaluate the efficacy of a smartphone-based reminder system in promoting pelvic floor muscle training (PFMT) to help postpartum women manage urinary incontinence (UI).

Methods. Forty-nine and 212 postpartum women in the intervention and control groups, respectively, received PFMT guidance using a leaflet and verbal instruction as the standard care at an obstetrics clinic in Japan. Women in the intervention group also received PFMT support using the smartphone-based reminder system between January and August 2014. For analysis, they were compared with historical controls between February 2011 and January 2012, who did not receive such support and were chosen by propensity score matching. The outcomes examined were PFMT adherence and UI prevalence. The former consisted of implementation rate (i.e., the percentage of women who reported performing PFMT during the intervention period), training intensity (i.e., the number of pelvic floor muscle contractions (PFMCs) per day), and training frequency (i.e., the number of days PFMT was performed per week); the latter consisted of selfreported UI prevalence at baseline and at the end of the 8-week intervention period.

Result. Propensity score matching resulted in 58 postpartum women ( $\mathrm{n}=29$ per group). The intervention group exhibited better PFMT adherence than the control group, in terms of PFMT implementation rate (69 vs. $31 \%, \mathrm{p}=0.008)$, median training intensity (15 vs. 1 PFMC reps/day, $\mathrm{p}=0.006)$, and training frequency ( 7 vs. 3 days/week, $\mathrm{p}<0.001)$. UI prevalence was not different between the groups at baseline, but was significantly reduced in the intervention group at 8 weeks $(0$ vs. $24 \%, p=0.004)$.

Conclusion. Our smartphone-based reminder system appears promising in enhancing PFMT adherence and managing postpartum UI in postpartum women. By enhancing PFMT adherence and improving women's ability to manage the condition, the reminder system could improve the health-related quality of life of postpartum women with UI.

Introduction

The International Continence Society (ICS) defines urinary incontinence (UI) as the "involuntary loss of urine." In a population-based study of countries with response rates $>60 \%$, the reported 
43 of Japanese women aged $\geq 40$ years revealed that $23 \%$ experienced UI at least once per week

44 (Homma et al., 2006). Pregnancy and childbirth are risk factors for UI (Wesnes et al., 2007;

45 Wesnes et al., 2010). The condition affects $20-35 \%$ of primiparous women following vaginal

46 delivery between the first and third months postpartum (Borello-France et al., 2006; Ekstrom et

47 al., 2008; Dinc et al., 2009). While it is considered a minor symptom in postpartum women, it

48 certainly could not be disregarded. UI is a major problem that lowers health-related quality of life

49 (HRQOL) (Goldberg et al., 2005) and may cause both social and hygiene problems.

50 Pelvic floor muscle training (PFMT) is considered the first-line conservative treatment for UI in

51 clinical practice guidelines in Japan (Minds 2013), the UK, (NICE 2015), and the USA (NGC

52 2006). The approach has been proven effective at treating and preventing postpartum UI

53 (Dumoulin et al., 2014). However, the efficacy of PFMT commencing specifically postnatally to

54 treat UI was only measured in three randomized controlled trials (RCTs), of which two were

55 small-scale studies of under 20 participants. Thus, further research is necessary.

56 Intensive PFMT, in which women are taught the exercises by physical therapists or specialists

57 and receive periodic follow-up on training frequency and efficacy, is reportedly better at

58 improving UI symptoms (Dumoulin et al., 2015). However, this approach is associated with high

59 costs; patients have to visit the hospital or clinic for follow-up or medical staff have to visit them

60 at home to provide guidance, and the approach is not covered by the healthcare systems of

61 several countries, including Japan. Moreover, one study involving Denmark women (mean age =

6254 years) found that although patients are instructed in intensive PFMT programs by

63 obstetricians, gynecologists, or urologists to adhere to the protocol to achieve the best results,

$64<50 \%$ visited the hospital for at least two-thirds of the scheduled follow-up appointments (Tibaek

$65 \&$ Dehlendorff, 2013).

66 Postpartum women, whose time may be otherwise occupied by childcare, would likely have more

67 difficulty in adhering to the protocols than would the women described in the aforementioned

68 study. However, few studies have investigated PFMT adherence in this population. Moreover,

69 postpartum women who lack the time to commit to intensive PFMT programs need a simple yet

70 free way to manage UI. To address this, we developed a smartphone-based reminder system to

71 provide PFMT support at home and enhance their adherence, thereby eliminating the need for

72 follow-up visits to their healthcare provider as in conventional intensive programs. 
73 In this study, we sought to clarify PFMT adherence in postpartum women and determine whether

74 a smartphone-based reminder system could help them manage UI. Our objective was to assess the

75 effectiveness of our PFMT support smartphone-based reminder system in helping women

76 manage postpartum UI.

\section{Materials \& Methods}

79 Study design

In this case-control study, postpartum women in the smartphone group, who received PFMT support via a smartphone-based reminder system, were compared with a historical control group, who did not use the reminder system. All participants received typical PFMT education administered by a midwife using a leaflet and verbal instruction.

PFMT support using smartphone-based reminder system

86

To develop the smartphone-based reminder system, we used the Rtime ${ }^{\mathrm{TM}}$ system (NEUES Co., Japan) (Higashida et al., 2015), which is designed to help patients follow medication and hospital visit schedules and works on $\mathrm{iOS}^{\mathrm{TM}}$ or Android ${ }^{\mathrm{TM}}$ smartphone platforms. Approximately 950,000 yen (8,400 USD) was used to develop the system, and approximately 450,000 yen (4,000 USD) is needed for the maintenance. A Grant-in-Aid helped us pay the costs. To register their smartphones with the system, the participants used one of two ways that involved sending blank e-mails. After a blank e-mail was sent, the system sent a response with a URL that directed them to an initial registration webpage and another URL for a website where they were instructed to enter their background characteristics (age, body mass index (BMI) before pregnancy, weight gain during pregnancy, and their child's birth weight). Data were encrypted using SSL during sending and receiving to prevent leakage of personal information; the technology is commonly used by websites that handle personal or credit card information. The day after the initial registration, the system started sending PFMT reminder messages every day at 9 a.m. The reminder displayed the number of days that had passed since the start of the training and a message with an emoji encouraging the participants to train ("Remember your PFMT today!") (Fig 1, left). On the first day, the message also displayed a URL where the participants were asked to enter their current UI status by answering the following question: "Do you have 
103 involuntary loss of urine that is a social or hygiene problem?", which is based on the ICS

104 definition at the time (Abrams, 2012); the system sent e-mails every day for 8 weeks, i.e., a total

105 of 56 reminder messages. The day after the final reminder message, the system automatically sent

106 an e-mail with a URL where the participants were asked to enter PFMT adherence-related

107 information and current UI status. The websites for entering data were designed with pull-down

108 menus and radio buttons so that the participants could easily enter the needed information (Fig 1,

109 right). No participants withdrew their participation because of difficulty in using the system

110 during the study period. All data that the participants entered on the websites were stored on the

111 system's server. The data can be accessed by logging into a system management website; login

112 IDs and passwords were managed only by the researchers.

114 Participants

115 Smartphone group

116 Participants for the smartphone group were recruited from January to August 2014 at an obstetric

117 clinic in Osaka Prefecture, Japan, which performs approximately 600 deliveries per year. Posters

118 explaining the study were displayed inside the clinic, and women who visited the clinic for 1-

119 month postpartum examinations were invited to participate. The inclusion criteria included

120 having undergone vaginal delivery and having a smartphone. The exclusion criteria were history

121 of pelvic surgery and cerebral infarction, as well as current hypertension, diabetes, hemorrhage,

122 cystitis, neurological disease of the urinary system, chronic cough, and diuretic use. Women were

123 recruited in the clinic's waiting room during the 20- to 30-min wait for a 1-month postpartum

124 checkup. After confirming that the postpartum women had a smartphone and fulfilled the other

125 criteria, the study was explained verbally and in writing. A midwife, who was either part of the

126 research or clinic staff, explained to the postpartum women who agreed to participate how to

127 register with the system and also provided an explanation on how to accurately perform PFMT

128 using a leaflet.

129 After registering, the women began receiving PFMT reminder messages via the smartphone-

130 based reminder system. Eight weeks thereafter, they were sent an e-mail with a link to a website

131 with a questionnaire. Aside from answering the question "Did you do PFMT these eight weeks?"

132 they were asked to self-report their training intensity (i.e., the number of pelvic floor muscle 
133 contractions (PFMCs) they performed per day; PFMC reps/day), training frequency (i.e., the

134 number of days they performed PFMT per week; PFMT days/week), and their UI status.

\section{Control group}

136 Women in the historical control group had given birth at the same obstetric clinic and were asked 137 to participate in a previous, different research concerning postpartum PFMT adherence and UI 138 status between February 2011 and January 2012. The inclusion criterion was postpartum women 139 who had vaginal delivery; the exclusion criteria were the same as those in the smartphone group, 140 history of pelvic surgery and cerebral infarction, as well as current hypertension, diabetes,

141 hemorrhage, cystitis, neurological disease of the urinary system, chronic cough, and diuretic use.

142 Midwives employed by clinic explained the research (verbally and in writing), distributed the 143 questionnaire, and provided the same PFMT instruction as that in the smartphone group. The 144 participants who agreed to participate answered a similar initial questionnaire as the smartphone 145 group regarding their background characteristics and current UI status. After 8 weeks, another 146 questionnaire was sent by mail with the same question items as those in the smartphone group, 147 which asked the participants to self-report their training, intensity, frequency, and current UI 148 status.

150 Pelvic floor muscle training

151 Women in both the smartphone and control groups received the same training: a midwife used a

152 leaflet and provided instruction on how to perform PFMT. The leaflet contained information on 153 the functions of the female pelvic floor muscle group and postpartum UI and described five 154 essential points when performing PFMT. Using a sagittal cross-section diagram of the female 155 pelvic area, the midwife explained how the pelvic floor muscle group supports the pelvic organs 156 and prevents UI from occurring when functioning normally. Subsequently, she explained how the 157 pelvic floor muscle group is damaged by pregnancy and childbirth and how incontinence can 158 occur if the damage is unaddressed. In addition, PFMT included the following five points: (1) 159 relax the body when performing the training, (2) slowly clench the vagina and anus as strongly as 160 possible without flexing the abdomen, (3) keep the pelvic floor muscles clenched while counting 161 up to 3-6, (4) perform 3 sets of 6 contractions every day (a total of 18 per day), and (5) continue 162 the training for at least 8 weeks. A diagram explaining the postures to be used during training 163 (standing, supine with the knees bent, prone with the elbows and knees on the floor, and standing 
164 while leaning on a table) was also employed. The method was based on Chiarelli's PFMT method

165 (Chiarelli et al., 2004), which has been deemed effective for antenatal and postpartum women in

166 a systematic review (Boyle et al., 2012).

168 Measurement

169 Participants provided the following information: age, BMI before pregnancy, weight gain during 170 pregnancy, and their child's birth weight. The participants referred to their maternal handbooks

171 for the latter two. Women in the intervention group provided these data via a website after 172 completing their initial registration in the smartphone-based reminder system. Women in the 173 control group wrote this information on the first questionnaire, which assessed the women's 174 background characteristics and UI status; after 8 weeks, they were sent another questionnaire 175 with the same question items as those in the smartphone group.

176 Our adherence outcomes included PFMT implementation rate (calculated as the percentage of 177 postpartum women who responded that they performed PFMT during the 8-week intervention 178 period) and median training intensity and frequency. UI prevalence at baseline and at 8 weeks 179 was assessed by the following question: "Do you have involuntary loss of urine that is a social or 180 hygiene problem?", and was calculated as the percentage of women answering "yes" to the 181 question. Women in the smartphone group responded via the website; women in the control 182 group responded through the questionnaire at baseline and 8 weeks thereafter.

184 Ethical considerations

185 The participants provided consent to participate in the study and to have their data published after 186 the objectives, methods, expected results, research cooperation benefits, and disadvantages were 187 explained to them. We also explained that not participating would not put the participant at a

188 disadvantage to ensure that their cooperation is not forced. Moreover, individuals in the 189 smartphone group provided a written consent to participate in the study. Agreement of women in 190 the control group to participate was confirmed by the answered questionnaire. This research was 191 approved by Osaka University Medical Science Department of Health Ethics Committee 192 (approval number: 151, 268). 
194 Statistical analysis

195 Propensity scores were calculated to adjust for the background characteristics of the smartphone 196 and control groups, which was followed by matching. Age, parity, and BMI (each being 197 considered a risk factor for UI) were considered predictor variables to calculate propensity 198 scores. Match tolerance was set as perfect agreement.

199 Continuous variables are expressed as median values with interquartile range; categorical 200 variables are expressed as number of cases with percentage. Background characteristics of the 201 smartphone group were compared with those of the control group using the Mann-Whitney U202 test for continuous variables and Fisher's exact test for categorical variables. Median values for 203 PFMC reps/day and PFMT days/week were calculated and tested after Bonferroni correction for 204 multiple comparisons. UI prevalence was directly evaluated using binomial distribution, as the 205 only possible values were 0 and 1 . All tests were two-sided, with $\mathrm{p}<0.05$ considered to indicate 206 statistical significance. Statistical analysis was conducted using SPSS version 24 (IBM Japan, 207 Inc.).

\section{Results}

210 Fig 2 showed flow diagram of postpartum women participating in the study. Forty-nine women 211 who met the inclusion criteria were included in the smartphone group. However, two women did 212 not record their current UI status the day after the initial registration and 16 women did not after

2138 weeks, respectively, and thus were excluded. Data of the remaining 31 women were analyzed.

214 The control group initially consisted of 212 women who returned the initial questionnaire.

215 However, eight were excluded for having had a cesarean section, one for history of cystitis, seven

216 for incomplete responses, and 75 for failing to return the second questionnaire at 8 weeks. Thus,

217 data of 121 women in the control group were included in the analysis. Propensity score matching 218 (PSM) of the two groups resulted in 29 matched pairs. The groups' background characteristics 219 before and after PSM are shown in Table 1. No significant differences in any background 220 characteristics either before or after PSM were observed.

221 Table 2 shows the PFMT implementation rate for the 8-week intervention period, the PFMC 222 reps/day and PFMT days/week of women who self-reported PFMT performance, and UI 223 prevalence at baseline and at 8 weeks. PFMT implementation rate was significantly higher in the 
224 smartphone group (69 vs. 31\%, p = 0.008). Median values for PFMC reps/day and PFMT

225 days/week were both significantly higher in the smartphone group (15 vs. $1 \mathrm{PFMC} /$ day, $\mathrm{Z}=$

$226-3.002, \mathrm{p}=0.006)(7$ vs. 3 days/week, $\mathrm{Z}=-4.144, \mathrm{p}<0.001)$. Among those who reported UI at

227 baseline, three women in the smartphone group and three out of eight women in the control group

228 experienced remission after 8 weeks. In addition, two women had a newly developed UI in the

229 control group, while no new cases were observed in the smartphone group. No difference in UI

230 prevalence at baseline (10 vs. $28 \%, \mathrm{p}=0.056)$ was noted, but UI prevalence was significantly

231 lower in the smartphone group at 8 weeks ( 0 vs. $24 \%, p=0.004)$. Compared with the control

232 group, the smartphone group exhibited better PFMT adherence, in terms of PFMT

233 implementation rate and median training intensity and training frequency. UI prevalence was not

234 different between the groups at baseline, but was significantly reduced in the smartphone group at

2358 weeks.

236

237 Discussion

238 In this study, postpartum women who received PFMT support via a smartphone-based reminder

239 system had a higher PFMT implementation rate during the intervention period, higher PFMC

240 reps/day, higher PFMT days/week, and improved UI prevalence after the 8-week intervention

241 period compared with postpartum women who only received a verbal instruction for PFMT.

242 We adopted propensity score matching to compare observational data with historical controls.

243 This technique allows us to analyze the data as a quasi-randomized controlled trial (Qin et al.,

244 2010; Haukoos et al., 2015). Our participants were not randomly selected. The intervention group

245 consisted of women who consented to use the smartphone-based reminder system; thus, the risk

246 of selection bias was unavoidable. In addition, the intervention group had few subjects. However,

247 with PSM, we were able to minimize problems related to cost and time and avoid the need for

248 blinding, which is a difficult condition for PFMT interventions (Qin et al., 2010; Haukoos et al.

249 2015). PSM allowed us to evaluate the PFMT intervention's effects on UI management using

250 groups with uniform background characteristics, while adjusting for the confounding risk factors

251 of age, parity, and BMI.

252 Any smartphone-based interventions have not been previously developed for UI. A previous

253 study where women were coached by a physical therapist throughout four sessions of PFMT

254 found that significantly more women in the intervention group performed PFMT in the previous 
255 month at 12 months after delivery (89 vs. 65\% in control) (Wilson \& Herbison, 1998). Another

256 study performed an intervention where postpartum women received three PFMT instructional

257 sessions at home administered by a midwife, health visitor, or continence adviser. The authors

258 found that 12 months after delivery, women in the intervention group had significantly higher

259 rates of performing PFMT in the previous month (79 vs. 48\% in control), with significantly more

260 PFMCs per day (20 vs. 5 in control) (Glazener et al., 2001). Our study demonstrated that PFMT

261 adherence could be enhanced by simply adding a smartphone-based reminder system to a

262 midwife-led PFMT instruction program, which has a similar efficacy to that of intensive PFMT

263 protocols where women have to visit a medical facility for follow-up or are visited by a

264 professional at home to receive coaching. Accordingly, the smartphone-based reminder system

265 excels as a simple yet free way to assist postpartum women in performing PFMT in their free

266 time at home. However, the withdrawal rate in the smartphone group was $36.7 \%$ (18/49). Boyle

267 \& Hay-Smith (2012) reported that the withdrawal rate of intensive PFMT in antenatal and

268 postnatal women was $4 \sim 38 \%$. We could not follow-up to clarify the reasons for withdrawal as

269 there was no response, even when sending a message to record the UI status and PFMT

270 adherence. Few studies have described the withdrawal rate of PFMT. In this way, our results are

271 valuable as they provide important data on this point, but we must consider the possibility of

272 detection bias.

273 Mørkved \& Bo (1997) also ran an 8-week intervention study on PFMT beginning after childbirth.

274 Women in their intervention group received individual coaching from a physical therapist to

275 ensure correct PFMC and received advice on how to continue the training at home. UI prevalence

276 was not different between the intervention and control groups at baseline; after 8 weeks, however,

277 a significant between-group difference was observed (intervention, 14.4\% [14/99]; control,

278 28.3\% [22/99]). Apparently, for PFMT, both conventional intensive programs and our

279 smartphone-based protocol could enhance adherence and lower UI occurrence. Moreover, our

280 research findings suggested that the smartphone-based reminder system allows patients to

281 successfully manage their UI, thereby helping them eliminate the symptoms and prevent its onset.

282 By contrast, other studies reported that intensive PFMT interventions are ineffective at reducing

283 UI incidence (Sleep \& Grant, 1987; Ewings et al. 2005). A recent study investigated PFMT

284 support provided by a physical therapist over the internet for three months. The study reported

285 that while the intervention group perceived significant improvement in its condition and reported

286 significantly reduced usage of incontinence aids compared with that at baseline, no significant 
287 differences were reported in the control group regarding the use of the postal support alone

288 (Sjostrom et al., 2013). One possible reason for such discrepancies could be the variable training 289 contents and evaluation periods from study to study. Hence, more studies on PFMT with a careful 290 selection of training contents and techniques for managing UI are necessary.

291 This study has two limitations. First, other factors that influence UI, PFMT implementation rate, 292 and PFMT adherence may remain undiscovered. Thus, failure to adjust for unobserved 293 confounders in the PSM model was possible. Hence, further clarification is necessary. Second, 294 our research was a single-institution, non-randomized observational study. Our intervention's 295 effectiveness needs to be further evaluated using a multi-center, large-scale research design.

296 The Japanese health care system does not offer rehabilitation aimed at recovering pelvic floor 297 muscle performance in postpartum women. Postpartum women only receive verbal instruction 298 regarding PFMT, which is the conservative treatment for urinary incontinence. Postpartum 299 women develop UI from diminished pelvic floor muscle function following pregnancy and 300 childbirth. Despite the fact that some women experience decreased HRQOL as a result (Takaoka 301 et al., 2017), the country has not actively introduced health care reforms or offered related 302 rehabilitation treatments, which could be attributed to costs and issues with ensuring adequate 303 staffing. Our smartphone-based reminder system could eliminate these challenges and, if widely 304 adopted, may improve the HRQOL of postpartum women with UI by enhancing PFMT 305 adherence and improving their ability to self-manage the condition.

\section{Conclusions}

308 Our smartphone-based reminder system appears promising in enhancing PFMT adherence and 309 managing postpartum UI in postpartum women. By enhancing PFMT adherence and improving women's ability to manage the condition, the reminder system could improve the health-related quality of life of postpartum women with UI.

\section{Acknowledgements}

314 We are grateful to all the women who participated in the study. We also thank Dr. Hiroaki

315 Jikihara, Director of Jikihara Women's Clinic; Chief Nurse Kayoko Furusawa; and the clinic's 316 midwives. 
318

319

320

321

322

323

324

325

326

327

328

329

330

331

332

333

334

335

336

337

338

339

340

\section{Reference}

Abrams P, Cardozo L, Khoury S, Wein AJ, and International Continence S. 2012. Incontinence: 5th International Consultation on Incontinence, Paris, February 2012. [Paris]: ICUD-EAU.

Borello-France D, Burgio KL, Richter HE, Zyczynski H, Fitzgerald MP, Whitehead W, Fine P, Nygaard I, Handa VL, Visco AG, Weber AM, Brown MB, Pelvic Floor Disorders N. 2006. Fecal and urinary incontinence in primiparous women. Obstetrics \& Gynecology 108:863872.

Boyle R, Hay-Smith EJ, Cody JD, Mokved S. 2012. Pelvic floor muscle training for prevention and treatment of urinary and faecal incontinence in antenatal and postnatal women. Cochrane Database of Systematic Reviews: John Wiley \& Sons, Ltd.

Chiarelli P, Murphy B, Cockburn J. 2004. Promoting urinary continence in postpartum women: 12-month follow-up data from a randomised controlled trial. International Urogynecology Journal and Pelvic Floor Dysfunction 15:99-105. DOI: 10.1007/s00192-004-1119-y.

Dinc A, Kizilkaya BN, Yalcin O. 2009. Effect of pelvic floor muscle exercises in the treatment of urinary incontinence during pregnancy and the postpartum period. International Urogynecology Journal and Pelvic Floor Dysfunction 20:1223-1231. DOI: 10.1007/s00192-009-0929-3.

Dumoulin C, Hay-Smith EJ, Mac Habee-Seguin G. 2014. Pelvic floor muscle training versus no treatment, or inactive control treatments, for urinary incontinence in women. [Update of Cochrane Database Syst Rev. 2010;(1):CD005654; PMID: 20091581]. Cochrane Database of Systematic Reviews 5:CD005654. DOI: 10.1002/14651858.CD005654.pub3.

Dumoulin C, Hay-Smith EJ, Frawley H, McClurg D, Alewijnse D, Bo K, Burgio K, Chen SY, Chiarelli P, Dean S, Hagen S, Herbert J, Mahfooza A, Mair F, Stark D, Van Kampen M, 
341 International Continence S. 2015. 2014 consensus statement on improving pelvic floor 342 muscle training adherence: International Continence Society 2011 State-of-the-Science 343 Seminar. Neurourology \& Urodynamics 34:600-605. https://dx.doi.org/10.1002/nau.22796.

344 Dumoulin C, Lemieux MC, Bourbonnais D, Gravel D, Bravo G, Morin M. 2004. Physiotherapy

345 for persistent postnatal stress urinary incontinence: a randomized controlled trial.

$346 \quad$ Obstetrics \& Gynecology 104:504-510. DOI: 10.1097/01.aog.0000135274.92416.62.

347 Ekstrom A, Altman D, Wiklund I, Larsson C, Andolf E. 2008. Planned cesarean section versus

348 planned vaginal delivery: comparison of lower urinary tract symptoms. International

349 Urogynecology Journal and Pelvic Floor Dysfunction 19:459-465. DOI: 10.1007/s00192-

$350 \quad$ 007-0461-2.

351 Ewings P, Spencer S, Marsh H, O'Sullivan M. 2005. Obstetric risk factors for urinary

352 incontinence and preventative pelvic floor exercises: cohort study and nested randomized

353 controlled trial.[Erratum appears in J Obstet Gynaecol. 2005 Nov;25(8):834-5]. Journal of

$354 \quad$ Obstetrics \& Gynaecology 25:558-564.

355 Glazener CM, Herbison GP, Wilson PD, MacArthur C, Lang GD, Gee H, Grant AM. 2001.

356 Conservative management of persistent postnatal urinary and faecal incontinence:

357 randomised controlled trial. British Medical Journal 323:593-596. DOI:

$358 \quad 10.1136 /$ bmj.323.7313.593.

359 Goldberg RP, Kwon C, Gandhi S, Atkuru LV, Sand PK. 2005. Urinary incontinence after multiple 360 gestation and delivery: impact on quality of life. International Urogynecology Journal and 361 Pelvic Floor Dysfunction 16:334-336. DOI: 10.1007/s00192-004-1252-7.

362 Haukoos JS, Lewis RJ. 2015. The propensity score. JAMA 314:1637-1638. DOI:

$363 \quad$ 10.1001/jama.2015.13480. 
364 Higashida Y, Sudou M, Kinouchi K, Ohashi K. 2015. Development of two-way communication 365 system using a mobile phone for pregnant women: acceptance of the system and usefulness 366 for the monitoring of passive smoking status. Nursing Journal of Osaka University 21:1-6 $367 \quad$ (Japanese).

368 Homma Y, Yamaguchi O, Hayashi K, and Neurogenic Bladder Society C. 2006. Epidemiologic 369 survey of lower urinary tract symptoms in Japan. Urology 68:560-564. DOI:

$370 \quad$ 10.1016/j.urology.2006.03.035

371 Morkved S, Bo K. 1997. The effect of postpartum pelvic floor muscle exercise in the prevention 372 and treatment of urinary incontinence. International Urogynecology Journal and Pelvic $373 \quad$ Floor Dysfunction 8:217-222.

374 Qin R, Titler MG, Shever LL, Kim T. 2010. Estimating effects of nursing intervention via 375 propensity score analysis. Nursing Research 57:444-452. DOI:

$376 \quad$ 10.1097/NNR.0b013e31818c66f6.

377 Sjostrom M, Umefjord G, Stenlund H, Carlbring P, Andersson G, Samuelsson E. 2013. Internet378 based treatment of stress urinary incontinence: a randomised controlled study with focus on pelvic floor muscle training. BJU International 112:362-372. DOI: 10.1111/j.1464-

381 Sleep J, Grant A. 1987. Pelvic floor exercises in postnatal care. Midwifery 3:158-164.

Takaoka S, Kondo Y, Kobayashi, Taniguchi T. 2017. Evaluation of the relationship between postpartum lower urinary tract symptoms and QOL: examination using the comprehensive questionnaire, SF-12ver.2. Journal of Japan Academy of Midwifery 30:78-87 (Japanese) gynaecologists and urologists at hospitals complete a pelvic floor muscle training 
389 Wesnes SL, Hunskaar S, Bo K, Rortveit G. 2010. Urinary incontinence and weight change during pregnancy and postpartum: a cohort study. American Journal of Epidemiology 172:10341044. DOI: $10.1093 / \mathrm{aje} / \mathrm{kwq} 240$.

392 Wesnes SL, Rortveit G, Bo K, Hunskaar S. 2007. Urinary incontinence during pregnancy. Obstetrics \& Gynecology 109:922-928. DOI: 10.1097/01.AOG.0000257120.23260.00

394 Wilson PD, Herbison GP. 1998. A randomized controlled trial of pelvic floor muscle exercises to treat postnatal urinary incontinence. International Urogynecology Journal and Pelvic Floor Dysfunction 9:257-264 
Figure 1

Screenshots of PFMT reminder e-mail and input screen for participant characteristics.

(A) Screenshot of PFMT reminder e-mail. (B) Screenshot of input screen for participant characteristics.

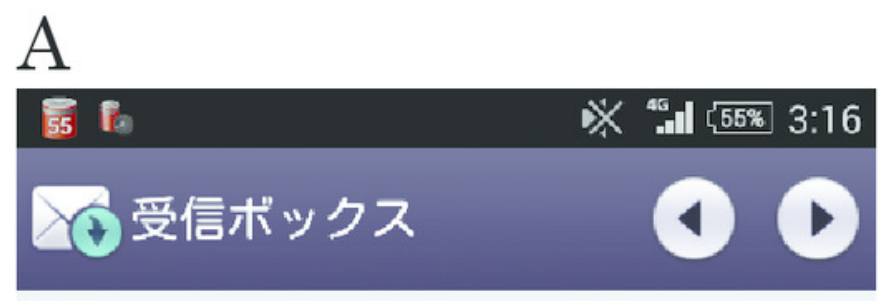

From info@rtime.jp

$$
2014 \text { 08/23 09:00 }
$$

Sub トレーニング2日目です

今日もトレーニングをがんばりましょう・ !... $\left.{ }^{\circ} \square^{\circ}\right) \times\left({ }^{\circ} \square^{\circ}\right)$ !

昨日の骨盤底筋訓練実施回数の日誌入力は こちらです。

$\downarrow \downarrow \downarrow \downarrow \downarrow \downarrow \downarrow \downarrow \downarrow \downarrow \downarrow \downarrow$

https://www.rtime.jp/chiken/sp/ou-nyshik kin/kainyu-qd/?p=1408599269179123\&mi $\underline{d}=34235$
$\mathrm{B}$

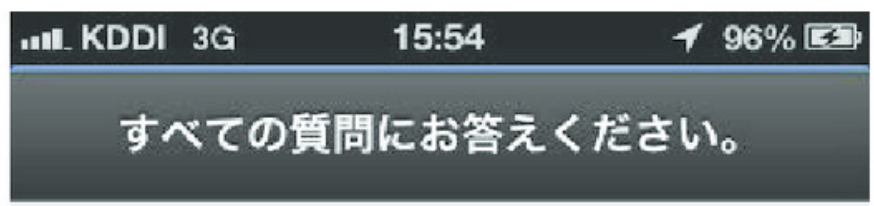

\section{*は必須入力項目です}

問1.あなたは、現在、以下の病気にかか

\section{っていますか？*}

0 病気底U

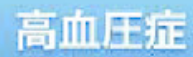

期层病

\section{脱挭塞/畄出血}

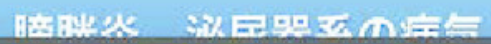

Copyright 2011-2013, NEUES Corp.

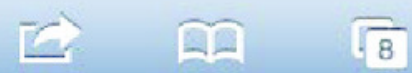




\title{
Figure 2
}

\section{Flow diagram of postpartum women participating in the study.}

\author{
[Historical control group] [Smartphone group]
}

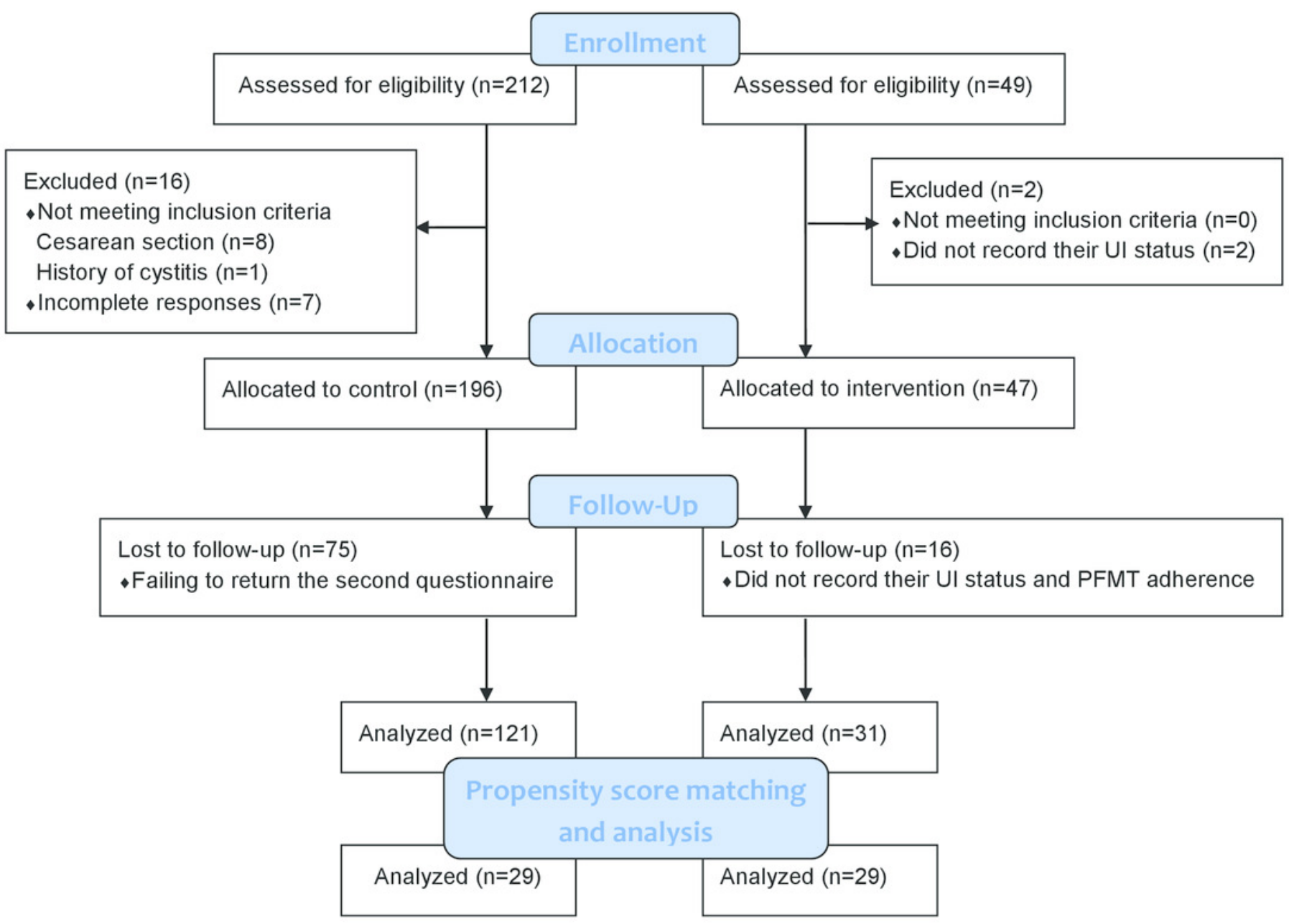




\section{Table 1 (on next page)}

Characteristics of smartphone and control groups before and after propensity score matching . 
Characteristics of smartphone and control groups before and after propensity score matching.

\begin{tabular}{|c|c|c|c|c|c|c|}
\hline & \multicolumn{3}{|l|}{ Before PSM } & \multicolumn{3}{|l|}{ After PSM } \\
\hline & $\mathrm{n}=31$ & $n=121$ & & $n=29$ & $\mathrm{n}=29$ & \\
\hline Age (years) & $34(31-36)$ & $33(30-36)$ & $0.476^{1)}$ & $34(31-36)$ & $34(32-37)$ & $0.760^{1)}$ \\
\hline Primipara & $7(22.6)$ & 42 (34.7) & $0.281^{2)}$ & $5(17.2)$ & $5(17.2)$ & $1.000^{21}$ \\
\hline Weight gain during pregnancy $(\mathrm{kg})$ & $10.0(9.0-12.0)$ & $9.0(7.4-11.0)$ & $0.067^{1)}$ & $10.0(8.5-11.8)$ & $9.5(8.0-12.0)$ & $0.691^{1)}$ \\
\hline Birth weight (g) & $3060 \quad(2749-3456)$ & $3150 \quad(2941-3361)$ & $0.203^{1)}$ & 3016 (2736-3349) & 3214 (3030-3368) & $0.106^{1)}$ \\
\hline
\end{tabular}

Data are shown as median (interquartile range) or number (\%).

PSM: propensity score matching, BMI: body mass index

${ }^{1)}$ Mann-Whitney U test

${ }^{2)}$ Fisher's exact test 


\section{Table 2 (on next page)}

Comparison of urinary incontinence and pelvic floor muscle training adherence measures between smartphone and control groups after propensity score matching. 
Comparison of urinary incontinence and pelvic floor muscle training adherence measures between smartphone and control groups after propensity score matching.

\begin{tabular}{lll}
\hline Smartphone & Control & $\mathrm{p}$ \\
$\mathrm{n}=29$ & $\mathrm{n}=29$ & \\
\hline
\end{tabular}

PFMT adherence

$\begin{array}{llllr}\text { Implementation rate } & 20(69.0) & 9 & (31.0) & 0.008^{1) *} \\ \text { Training intensity (PFMC reps/day) } & 15(3-18) & 1 & (1-4) & 0.006^{1) *} \\ \text { Training frequency (PFMT days/week) } & 7(6-7) & 3(1-4) & <0.001^{1) *}\end{array}$

UI prevalence

\begin{tabular}{|c|c|c|c|}
\hline Baseline & 3 (10.3) & 8 (27.6) & $0.056^{2)}$ \\
\hline After 8 weeks & $0 \quad(0.0)$ & $7 \quad(24.1)$ & $0.004^{2) *}$ \\
\hline
\end{tabular}

Data are shown as median (interquartile range) or number (\%).

PFMT: pelvic floor muscle training, PFMC: pelvic floor muscle contraction, UI: urinary incontinence

${ }^{1)}$ Bonferroni correction followed by Mann-Whitney $U$ test

${ }^{2)}$ Binomial test

*Significant difference 